From the Department of Neurosurgery at Riverside University Health System in Moreno Valley, California and Western University, College of

Osteopathic Medicine, Department of Neurosurgery,

Pomona, California.

Financial Disclosures: None reported.

Support: None reported.

Address correspondence to

James A. D. Berry, DO,

Riverside University Health

System, 26520 Cactus

Avenue, Moreno Valley,

California 92555-3927.

Email:

jamesberrydo@gmail.com

Submitted

September 22, 2018;

revision received

May 6, 2019;

accepted

August 14, 2019.

\section{Pathophysiologic Mechanisms of Concussion, Development of Chronic Traumatic Encephalopathy, and Emerging Diagnostics: A Narrative Review}

James A. D. Berry, DO; Christopher Elia, DO; Raed Sweiss, DO; Shokry Lawandy, DO Ira Bowen, DO; Bailey Zampella, DO; Harneel Saini, OMS IV; James Brazdzionis, DO; Dan Miulli, DO

Pathophysiological mechanisms and cascades take place after a mild traumatic brain injury ( $\mathrm{mTBI}$ ) that can cause long-term sequelae, including chronic traumatic encephalopathy in patients with multiple concurrent TBIs. As diagnostic imaging has become more advanced, microanatomical changes present after $\mathrm{mTBI}$ may now be more readily visible. In this narrative review, the authors discuss emerging diagnostics and findings in mTBI through advanced imaging, electroencephalograms, neurophysiologic processes, Q2 biochemical markers, and clinical tissue tests in an effort to help osteopathic physicians to understand, diagnose, and manage the pathophysiology behind $\mathrm{mTBI}$, which is increasingly prevalent in the United States.

J Am Osteopath Assoc. 2020;120(9):582-589. Published online August 12, 2020. doi:10.7556/jaoa.2020.074

Keywords: cerebral edema, cognitive stimulation, concussion, glymphatics, optic nerve sheath diameter, traumatic brain injury

nderstanding and recognition of concussion has improved over the past 2 decades, resulting in a substantial increase in the number of diagnosed cases and increasing the known incidence. The increased awareness among physicians of the clinical criteria has led to a dramatic increase in the number of cases diagnosed annually. The Centers for Disease Control and Prevention have declared this a public health crisis, with an estimated 3.8 million sports- and recreation-related concussions reported annually in the United States. ${ }^{1}$ Traumatic brain injury (TBI) is a spectrum of injury severity, ranging from severe to mild, with "severe" defined as a Glasgow Coma Scale of 8 or less and with "mild" including transient impairment of cognition and or consciousness. Although a concussion is clinically defined as a mild traumatic brain injury (mTBI), it can have long-term consequences. Previously, concussion was thought to be a clinical diagnosis based only on criteria of negative diagnostic imaging in the context of head trauma with associated confusion, amnesia, or loss of consciousness. There is now objective diagnostic laboratory and radiographic evidence proving that concussion is more than just a clinical diagnosis. The medical profession is starting to understand the underlying pathophysiologic mechanisms and the long-term consequences of repeated mTBIs. The long-term repercussions of successive concussions have an escalating detrimental pathologic effect. Concussion and more severe forms of mTBI constitute a continuous spectrum of severity, with chronic traumatic encephalopathy (CTE) being a 
consequence of these injuries. The long-term consequences can cause life-altering chronic changes that interfere with a patient's social, academic, and occupational performance. We discuss and summarize the emerging diagnostics and findings involved in mTBI and CTE through advanced imaging, electroencephalograms (EEGs), neurophysiologic processes, biochemical markers, and clinical tissue tests.

\section{Concussion and TBI}

Concussion is the consequence of head impact, the force of initial contact, and the linear and rotational inertial acceleration-deceleration injury. Rapid rotational head movements generate shear forces throughout the brain, which can produce cavitation and disruptions at a cellular level. ${ }^{2}$ Percussive shockwaves transmit forces through tissues of different densities that are absorbed in the cerebrospinal fluid (CSF), gray matter, and white matter. ${ }^{3}$ The ventricles appear to dampen and absorb some kinetic energy, providing some partial protection to the brain. ${ }^{4}$ The mechanical energy transmitted throughout the tissue of the cerebrum is transferred into the cells, disrupting cytoskeletal architecture, impairing organelle function, and affecting biochemical activity. ${ }^{5,6}$ This cascade disrupts the production and release of neurotransmitters and can interfere with the proper functioning of cell surface receptors. ${ }^{7,8}$ Furthermore, there is an early excessive release of neurotransmitters, such as glutamate, which can cause an excitatory neuronal toxicity. ${ }^{9,10}$ The result is aberrations of neuronal electrochemical signal transduction and synchrony between communicating neurons. ${ }^{11}$ TBI further impairs long-term signal transduction, as damaged oligodendrocytes cannot produce enough myelin to properly insulate damaged axons. ${ }^{12}$ White matter tract disruption is influenced by the directional plane (sagittal, coronal, axial) through which the rotational force is applied. ${ }^{13}$ Proximal axonal injuries closer to the soma can recover faster than more distal injuries. ${ }^{14}$ Fluid-attenuated inversion recovery magnetic resonance imaging (MRI) sequencing is effective in detecting subtle fluid changes deep in the brain's parenchyma. Delayed imaging in patients with mTBI reveals evidence of cytotoxic and vasogenic edema in patients after concussion. While damage to white matter tracts are not immediately visible in acutely concussed patients, pathologic changes are apparent on magnetic resonance angiography (MRA) diffusion tensor imaging (DTI) several months after the injury, even if the patient no longer has symptoms. ${ }^{15}$

The impact force transmits rapid changes throughout the central nervous system (CNS), altering the function of neurons and astrocytes and disrupting the blood-brain barrier (BBB) in vivo. ${ }^{16}$ In TBI, nicotinamide adenine dinucleotide phosphate oxidase 4 , which is a phagocytetype oxidase enzyme responsible for producing reactive oxidant species, causes autotoxicity and breakdown of the BBB, further disrupting cerebral function. ${ }^{17,18}$ The injured brain has a significant increase in metabolic demands that can affect energy balance; thus, a transient state of hyperglycolysis occurs because of a consumption of nutrients, vital molecules, and intracellular energy stores, while complex biochemical cascades are initiated. ${ }^{19}$ The ability of the CNS to regulate blood flow, clear metabolic waste byproducts, protect against hyperexcitable states, and carry out routine physiologic function is impaired after TBI. ${ }^{19}$ Many of these compensatory responses can add detrimental secondary brain damage, such as the harmful effects of the inflammatory cascade. ${ }^{19}$

The cells of the hippocampal formation appear to be particularly susceptible to the effects of rotational shear strain injury, leading to both the retrograde and anterograde amnesia so common after mTBI. ${ }^{19}$ In patients who have experienced TBI with loss of consciousness, MRA DTI has shown pathologic changes in the reticular activating system, which is responsible for alertness and consciousness. ${ }^{20}$ The timing of the initial cognitive impairment or confusion may be explained by neuronal plasma membrane permeability disruption. This disruption typically begins to rapidly recover after mTBIs, in about 10 to 15 minutes, when ionic electrochemical membrane gradients are restored. ${ }^{21}$ It is no coincidence 
that this correlates to the improvement time in many mTBIs that have confusion as the only symptom.

Quantitative EEG can identify unusual patterns of brain oscillations in patients with mTBI both during sleep and awake states. Such brain oscillations lead to abnormal sleep patterns and difficulties with concentration. The concussed patient has less $\alpha$ wave activity and more $\theta$ and $\Delta$ wave activity while awake and a longer rapid eye movement stage while asleep. ${ }^{22,23}$ Quantitative EEG can detect changes in patients with mTBI both immediately and a year later. ${ }^{24}$ The degree of observable aberrations present on EEG seems to correlate with the intensity of active symptoms. ${ }^{25,26}$

\section{Glymphatics $^{26}$}

Like blood, CSF conveys both brain chemicals and waste products. CSF circulation begins from the choroid plexus, and CSF is absorbed through arachnoid granulations. However, there is an additional CSF outflow tract that drains the paleocortex and medial temporal lobe. This outflow is found in the cribriform plate along the olfactory stria and olfactory bulb and drains into the nasal submucosa. TBI has been shown to reduce this system of drainage and reduce the clearance of brain chemical waste products. It has been believed for many centuries that the human brain does not have any other structural tissue to clear waste products, such as the lymphatics found throughout the rest of the body. Although brain lymphatics were first described in 1816 by the Italian anatomist Paolo Mascagni, this misperception of the lack of lymphatics lasted deep into the 20th century.

In the 21 st century, the use of 2-photon excitation microscopy in combination with fluorescent tracers was able to show the flow of CSF entering the perivascular spaces around penetrating arteries, exchanging with surrounding interstitial fluid. ${ }^{27}$ This indirectly showed the clearance of interstitial waste from the brain parenchyma, which is a primary function of lymphatics. $^{27}$ The Danish neuroscientist Maiken Nedergaard coined the term glymphatics, recognizing that this pathway was unique and different from the per- ipheral lymphatic system and acknowledging the functional participation of the glial cells in this process. ${ }^{28}$ The discovery of glymphatics demonstrated that the CNS was able to clear metabolic waste products, excess fluids, proteins, and other interstitial debris from the interstitial spaces of the cerebral parenchyma through CSF. However, extracting the waste from CSF remained elusive until structural meningeal lymphatic sinuses were discovered. ${ }^{29-31}$ Pulsatile CSF drives this paravascular fluid movement. ${ }^{32}$ Individual perivascular astrocyte water channels, known as aquaporins, directly participate in the glymphatic pathway, ${ }^{33}$ with astrocytic aquaporin 4 receptor as essential for the interstitial fluid-CSF exchange. ${ }^{33,34}$ The glymphatic system responds to different electromagnetic fields produced by the brain, mostly during slow-wave sleep. ${ }^{35}$ The prevalence of sleep $\Delta$ waves below $4 \mathrm{~Hz}$ and $\theta$ waves at 4 to $7 \mathrm{~Hz}$, seen more in patients with mTBI, were associated with the highest rate of glymphatic clearance of waste. The normal synchronicity during awake and sleep cycles is lost with concussion, as demonstrated on quantitative EEG, leading to decreased clearance function of CSF and glymphatics and the continued buildup of waste products. ${ }^{35}$

The glymphatic system clears proteins and small lipophilic molecules during sleep, including amyloid $\beta$ protein. ${ }^{36,37}$ The accumulation of amyloid $\beta$ protein has been observed in patients with Alzheimer disease and CTE, ${ }^{38}$ but treatments can enhance the clearance of CSF and glymphatics, which may then lead to recovery. Several studies have demonstrated that patients with a history of concussion or other TBI have a significantly higher risk of Alzheimer disease compared with the rest of the population. ${ }^{39}$ The administration of intrathecal gadolinium to assess glymphatic function by MRI is currently being investigated in concussion and idiopathic normal-pressure hydrocephalus. ${ }^{40,41}$

\section{CTE and Other Chronic Manifestations of Repeated Concussive mTBI}

CTE is the clinical manifestation of chronic neurodegenerative pathologic changes from multiple prior 
concussive mTBIs. Cerebral autopsy specimens from CTE patients demonstrate cellular deposition of amyloid $\beta$ protein, hyperphosphorylated $\tau$ protein in neurofibrillary tangles, astrocytic tangles, and increased neurites around cortical small blood vessels. ${ }^{42}$ Alzheimer disease and CTE are distinct entities but have many overlapping characteristics, including gross and micropathologic features, clinical presentations, and risk factors. ${ }^{43}$

Neurodegenerative changes in the cells of the pars compacta of the substantia nigra and ventral tegmental area in the midbrain of patients who have experienced multiple mTBIs can result in parkinsonian complex movement disorders. ${ }^{44}$ Positron emission tomography scans show decreased uptake of glucose-tagged metabolites in the pars compacta of the midbrain in former boxers who developed parkinsonian-type movement disorders. ${ }^{45}$ Accordingly, dementia pugilistica is a combination of neuropathologic changes causing cognitive impairment found in $20 \%$ of retired boxers. ${ }^{46}$ The brains of deceased CTE patients demonstrate extensive deposition of $\beta$ amyloid throughout the cerebrum at levels comparable to patients with advanced Alzheimer disease who were many decades older and which may be the result of chronic neuronal toxin buildup due to decreased glymphatic and CSF clearance, as well as to the forceful breakdown of the cell and axon cytoskeleton. ${ }^{47}$ Neurocognitive changes in boxers labeled as "punch drunk" over the past century were observed without understanding the seriousness of such injuries.

Preseason cognitive tests are essential to determine an athlete's baseline cognitive function before engaging in sports, because it is difficult for a physician to later adequately assess the change in a concussed athlete's neurocognitive performance. Multiple preseason baseline athlete cognitive tests are available. The 2 most commonly used are the Standardized Assessment of Concussion form and the Acute Concussion Evaluation form. ${ }^{48}$

While US football has received enormous media attention regarding concussion and $\mathrm{CTE},{ }^{48}$ the first observed pathologic changes, later termed CTE, were discovered in the brains of deceased boxers. ${ }^{48-50}$ Decades after punch-drunk boxers received diagnoses of dementia pugilistica, pathologists performed postmortem examinations on these boxers' brains. The gross pathologic specimens demonstrated cavum septum pellucidum, cerebral atrophy with dilated ventricles that were disproportionate to the patient's age, depigmentation of the substantia nigra, and fenestrations and scarring of the cerebellar tonsils. ${ }^{51}$ The micropathologic examination demonstrated neuronal loss within the cerebral cortex, substantia nigra and cerebellar tonsils, and neurofibrillary degeneration and senile plaques disproportionate to the patient's age. ${ }^{52,53}$

Neuropathologic analysis of boxers with severe memory disturbance, cognitive impairment, profound dysphoria, and Parkinsonian movement symptoms were found to have a high degree of neurofibrillary tangles, particularly in layers II and III of the neocortex. ${ }^{54}$ The pathologic consequences of CTE became more prominent in the media when, in the 21 st century, the disorder was discovered in professional US football players. ${ }^{48}$ In 2010, an autopsy on a 44-year-old National Football League athlete who had an apolipoprotein E genotype demonstrated diffuse cerebral tauopathy, neurofibrillary tangles, and neuritic threads. ${ }^{48}$ Similar autopsy results fueled a 2012 multi-billion-dollar lawsuit by the National Football League Players Association and drew attention to the disorder among the public.

It soon became apparent that, in recreational sports, a concussion could be sustained anytime a blow to the head occurred. ${ }^{55}$ As CTE awareness spread, the disorder received increased funding both from private and government sources. Interestingly, many patients with CTE and psychiatric disturbances had sustained injuries to some of the key neurotransmitter-producing nuclei and tracts projecting to the neocortex, such as the norepinephrine-producing locus ceruleus and serotoninproducing dorsal raphe nuclei. ${ }^{50,56}$

In investigating the imaging of these patients, $\mathrm{CT}$ and MRI findings of patients with acute concussive mTBI were negative for gross hemorrhages ${ }^{57}$; however, 
microscopic pathologic examination of postmortem patients with CTE demonstrate hemosiderin deposits. These deposits suggest evidence of a chronic microbleed. ${ }^{57}$

\section{Serum Biomarkers}

In 2018, the US Food and Drug Administration approved the first serum TBI marker test as an aid in the diagnosis of concussion mTBI. ${ }^{58}$ The test detects the presence of 2 proteins in the brain: ubiquitin carboxy-terminal hydrolase-L1 (UCH-L1) and glial fibrillary acid protein (GFAP). UCH-L1 is a neuronspecific protein that is present throughout the CNS and is believed to serve the antioxidant function of scavenging the brain for toxins, thereby decreasing long-term injury. ${ }^{59,60}$ GFAP is an intermediate glial cell filament protein in CNS astrocytes and the ventricular lining ependymal cells. ${ }^{61}$ GFAP provides cytoskeletal support and strength to the astrocyte. ${ }^{62,63}$ The delayed changes seen on MRA DTI after mTBI may be the result of decreased GFAP and resultant changes in the brain cytoskeleton. The intracellular UCH-L1 and GFAP proteins from the CNS are released into the bloodstream when a TBI has occurred and are detected by enzyme-linked immunosorbent assay within 1 hour of initial head impact. ${ }^{64-66}$ Aside from UCH-L1 and GFAP, other biomarkers are currently under investigation.

The calcium-binding protein $\mathrm{S} 100 \mathrm{~B}$ is a glialspecific protein found in the cytoplasm of astrocytes as the result of the breakdown of the $\mathrm{BBB}^{67-71}$; however, this biomarker is neither specific nor approved as a biomarker for TBI. Cytokine CCL11 is a small cytokine protein inflammatory marker that has been found to be elevated in the CSF of patients with CTE, but not in those with Alzheimer disease. ${ }^{71}$ Neurofilament light polpeptide is an intermediate filament found in the cytoskeleton of neurons and provides structural support to the axon. It has also been detected in the CSF of patients with TBI and is believed to provide a prognostic value. ${ }^{72}$ Patients who have had an mTBI often cannot remember the incident, and these serum biomarkers may help physicians to determine whether a patient has had an mTBI and to potentially quantify the severity of the injury. Such serum biomarkers require further studies and investigation.

\section{Conclusion}

Concussion was once thought to be strictly a clinical diagnosis with symptoms of confusion, amnesia, or loss of consciousness with no associated radiographic, biochemical, or pathologic criteria. New discoveries in biomarkers, imaging, and pathophysiology over the past few years are dramatically shaping how physicians diagnose and manage TBI and have led to an increased understanding and recognition of this disease. Physicians should not limit their focus on concussion to the sport of US football but understand that any sport with a potential for head impact can produce TBI. Raising awareness of the short- and long-term neurophysiologic ramifications of concussion is critical, using tests available to diagnose concussion as the first of a myriad of steps in caring for these patients. ${ }^{73}$ Although there is a scarcity of literature pertaining to the direct use of osteopathic manipulative treatment in patients with mTBI, there are a number of active investigational studies, including some currently in process at our institution. ${ }^{74}$ This article serves as a review for osteopathic physicians in the primary care setting who are seeing an increase in the number of patients presenting with concussions each year. Understanding of the emerging diagnostics that will be used to treat patients and to help improve clinical outcomes is just the beginning. The use of serum biomarkers to diagnose concussion will help osteopathic physicians effectively identify patients with mTBI and safely allow such patients return to play. Out of all the concussion biomarkers being researched, the current data demonstrate that GFAP has the highest specificity for acute brain injury. With only a few serum biomarkers having received the approval of the US Food and Drug Administration and more actively under investigation, future studies will be needed to determine the sensitivity and specificity of these serum biomarkers and their 
potential in not only achieving a diagnosis, but also prognostication.

\section{Author Contributions}

All authors provided substantial contributions to conception and design, acquisition of data, or analysis and interpretation of data; all authors drafted the article or revised it critically for important intellectual content; all authors gave final approval of the version of the article to be published; and all authors agree to be accountable for all aspects of the work in ensuring that questions related to the accuracy or integrity of any part of the work are appropriately investigated and resolved.

\section{References}

1. Faul M, Xu L, Wald MM, Coronado VG. Traumatic Brain Injury in the United States: Emergency Department Visits, Hospitalizations and Deaths 2002-2006. Centers for Disease Control and Prevention, National Center for Injury Prevention and Control; 2010. https://www. cdc.gov/traumaticbraininjury/pdf/blue_book.pdf. Accessed August 4 , 2020.

2. Goeller J, Wardlaw A, Treichler D, O'Bruba J, Weiss G. Investigation of cavitation as a possible damage mechanism in blast-induced traumatic brain injury. J Neurotrauma. 2012; J29(10):1970-1981. doi: 10.1089/ neu.2011.2224.

3. Thibault LE, Meaney DF, Anderson BJ, Marmarou A. Biomechanical aspects of a fluid percussion model of brain injury. $J$ Neurotrauma. 1992;9(4):311-322.

4. Ivarsson J, Viano DC, Lövsund P. Influence of the lateral ventricles and irregular skull base on brain kinematics due to sagittal plane head rotation. J Biomech Eng. 2002;124(4):422-431.

5. Miller WJ, Leventhal I, Scarsella D, Haydon PG, Janmey P, Meaney DF. Mechanically induced reactive gliosis causes ATP-mediated alterations in astrocyte stiffness. J Neurotrauma. 2009;26(5):789-797. doi: $10.1089 /$ neu.2008-0727

6. Zhang L, Rzigalinski BA, Ellis EF, Satin LS. Reduction of voltage-dependent Mg2+ blockade of NMDA current in mechanically injured neurons. Science. 1996;274(5294):1921-1923.

7. Kao CQ, Goforth PB, Ellis EF, Satin LS. Potentiation of GABA(A) currents after mechanical injury of cortical neurons. J Neurotrauma. 2004;21(3):259-270.

8. Spaethling JM, Klein DM, Singh P, Meaney DF. Calcium-permeable AMPA receptors appear in cortical neurons after traumatic mechanical injury and contribute to neuronal fate. J Neurotrauma. 2008;25 (10):1207-1216. doi: 10.1089/neu.2008.0532

9. Cantu $\mathrm{D}$, Walker $\mathrm{K}$, Andresen $\mathrm{L}$, et al. Traumatic brain injury increases cortical glutamate network activity by compromising GABAergic control. Cereb Cortex. 2015;25(8):2306-2320. doi: 10.1093/cercor/ bhu041

10. Lau A, Tymianski M. Glutamate receptors, neurotoxicity and neurodegeneration. Pflugers Arch. 2010;460(2):525-542. doi: 10.1007/ s00424-010-0809-1

11. Weber JT, Rzigalinski BA, Willoughby KA, Moore SF, Ellis EF. Alterations in calcium-mediated signal transduction after traumatic injury of cortical neurons. Cell Calcium. 1999;26(6):289-299.
12. Flygt J, Gumucio A, Ingelsson M, et al. Human traumatic brain injury results in oligodendrocyte death and increases the number of oligodendrocyte progenitor cells. J Neuropathol Exp Neurol. 2016;75 (6):503-515. doi: 10.1093/jnen/nlw025

13. Mychasiuk R, Hehar H, Candy S, Ma I, Esser MJ. The direction of the acceleration and rotational forces associated with mild traumatic brain injury in rodents effect behavioural and molecular outcomes. J Neurosci Methods. 2016;257:168-178. doi: 10.1016/j. jneumeth.2015.10.002

14. Lucas JH. Proximal segment retraction increases the probability of nerve cell survival after dendrite transection. Brain Res. 1987;425 (2):384-387.

15. Virji-Babul N, Borich MR, Makan N, et al. Diffusion tensor imaging of sports-related concussion in adolescents. Pediatr Neurol. 2013;48 (1):24-29. doi: 10.1016/j.pediatrneurol.2012.09.005

16. Stamatovic SM, Keep RF, Andjelkovic AV. Brain endothelial cell-cell junctions: how to "open" the blood brain barrier. Curr Neuropharmacol. 2008;6(3):179-192. doi: 10.2174/157015908785777210

17. Zhu P, Tong BM, Wang R, et al. Nox4-dependent ROS modulation by amino endoperoxides to induce apoptosis in cancer cells. Cell Death Dis. 2013;4:e552. doi: 10.1038/cddis.2013.68

18. Casas Al, Geuss E, Kleikers PWM, et al. NOX4-dependent neurona autotoxicity and BBB breakdown explain the superior sensitivity of the brain to ischemic damage. Proc Natl Acad Sci U S A. 2017;114 (46):12315-12320. doi: 10.1073/pnas.1705034114

19. Geddes DM, LaPlaca MC, Cargill RS 2nd. Susceptibility of hippocampal neurons to mechanically induced injury. Exp Neurol. 2003;184(1):420-427.

20. Ordóñez-Rubiano EG, Johnson J, Enciso-Olivera CO, et al. Reconstruction of the ascending reticular activating system with diffusion tensor tractography in patients with a disorder of consciousness after traumatic brain injury. Cureus. 2017;9(9):e1723. doi: 10.7759 /cureus. 1723

21. Geddes DM, Cargill RS 2nd, LaPlaca MC. Mechanical stretch to neurons results in a strain rate and magnitude-dependent increase in plasma membrane permeability. J Neurotrauma. 2003;20(10):1039-1049.

22. Khoury S, Chouchou F, Amzica F, et al. Rapid EEG activity during sleep dominates in mild traumatic brain injury patients with acute pain. J Neurotrauma. 2013;30(8):633-641.

23. Haneef Z, Levin HS, Frost JD Jr, Mizrahi EM. Electroencephalography and quantitative electroencephalography in mild traumatic brain injury. J Neurotrauma. 2013;30(8):653-656. doi: 10.1089/neu.2012.2585

24. Franke LM, Walker WC, Hoke KW, Wares JR. Distinction in EEG slow oscillations between chronic mild traumatic brain injury and PTSD. Int J Psychophysiol. 2016;106:21-29. doi: 10.1016/j.jpsycho.2016.05.010

25. Virji-Babul N, Hilderman CG, Makan N, et al. Changes in functional brain networks following sports-related concussion in adolescents. J Neurotrauma. 2014;31(23):1914-1919. doi: 10.1089/neu.2014.3450

26. Iliff JJ, Wang M, Liao Y, et al. Paravascular pathway facilitates CSF flow through the brain parenchyma and the clearance of interstitial solutes, including amyloid $\beta$. Sci Trans/ Med. 2012;4(147): 147ra111. doi: 10.1126/scitransImed. 3003748

27. Konnikova M. Goodnight. Sleep Clean. The New York Times. January 11, 2014. https://www.nytimes.com/2014/01/12/opinion/sunday/ goodnight-sleep-clean.html Accessed February 18, 2014. 
28. Louveau A, Smirnov I, Keyes TJ, et al. Structural and functional features of central nervous system lymphatic vessels. Nature. 2015;523(7560): 337-341. doi: 10.1038/nature14432

29. Aspelund A, Antila S, Proulx ST, et al. A dural lymphatic vascular system that drains brain interstitial fluid and macromolecules. J Exp Med. 2015;212(7):991-999. doi: 10.1084/jem.20142290

30. Kim SC, Park SW, Ryoo I, et al. Contrast-enhanced FLAIR (fluid-attenuated inversion recovery) for evaluating mild traumatic brain injury. PLoS One. 2014;9(7):e102229. doi: 10.1371/journal. pone. 0102229

31. Rennels ML, Gregory TF, Blaumanis OR, Fujimoto K, Grady PA Evidence for a 'paravascular' fluid circulation in the mammalian central nervous system, provided by the rapid distribution of tracer protein throughout the brain from the subarachnoid space. Brain Res 1985;326(1):47-63.

32. Amiry-Moghaddam M, Ottersen OP. The molecular basis of water transport in the brain. Nat Rev Neurosci. 2003;4(12):991-1001.

33. Verkman AS, Binder DK, Bloch O, Auguste K, Papadopoulos MC. Three distinct roles of aquaporin-4 in brain function revealed by knockout mice. Biochim Biophys Acta. 2006;1758(8):1085-1093.

34. Yool AJ. Aquaporins: multiple roles in the central nervous system. Neuroscientist. 2007;13(5):470-485.

35. Xie L, Kang $\mathrm{H}, \mathrm{Xu} \mathrm{Q}$, et al. Sleep drives metabolite clearance from the adult brain. Science. 2013;342(6156):373-377. doi: 10.1126/ science. 1241224

36. Rangroo Thrane V, Thrane AS, Plog BA, et al. (2013). Paravascular microcirculation facilitates rapid lipid transport and astrocyte signaling in the brain. Sci Rep. 2013;3:2582. doi: 10.1038/srep02582.

37. Mehler MF, Gokhan S. Mechanisms underlying neural cell death in neurodegenerative diseases: alterations of a developmentallymediated cellular rheostat. Trends Neurosci. 2000;23(12):599-605.

38. Mendez MF, Paholpak P, Lin A, Zhang JY, Teng E. Prevalence of traumatic brain injury in early versus late-onset Alzheimer's disease. J Alzheimers Dis. 2015;47(4):985-993. doi: 10.3233/JAD-143207

39. Plassman BL, Havlik RJ, Steffens DC, et al. Documented head injury in early adulthood and risk of Alzheimer's disease and other dementias. Neurology. 2000;55(8):1158-1166.

40. Eide PK, Ringstad G. MRI with intrathecal MRI gadolinium contrast medium administration: a possible method to assess glymphatic function in human brain. Acta Radiol Open. 2015;4 (11):2058460115609635. doi: 10.1177/2058460115609635

41. Ringstad G, Vatnehol SAS, Eide PK. Glymphatic MRI in idiopathic normal pressure hydrocephalus. Brain. 2017;140(10):2691-2705. doi: 10.1093/brain/awx191

42. McKee AC, Stein TD, Kiernan PT, Alvarez VE. The neuropathology of chronic traumatic encephalopathy. Brain Pathol. 2015;25(3):350-364. doi: $10.1111 / \mathrm{bpa} .12248$

43. Turner RC, Lucke-Wold BP, Robson MJ, Lee JM, Bailes JE. Alzheimer's disease and chronic traumatic encephalopathy: distinct but possibly overlapping disease entities. Brain Inj. 2016;30 (11):1279-1292. doi: 10.1080/02699052.2016.1193631

44. Stern MB. Head trauma as a risk factor for Parkinson's disease. Mov Disord. 1991;6(2):95-97.

45. Bhidayasiri R, Chotipanich C, Joutsa J, et al. Boxing and Parkinson disease: a link or a myth? An 18F-FDOPA PET/CT study in retired
Thai traditional boxers. Parkinsonism Relat Disord. 2012;18

(5):694-696. doi: 10.1016/j.parkreldis.2012.01.010

46. Kokjohn TA, Maarouf CL, Daugs ID, et al. Neurochemical profile of dementia pugilistica. J Neurotrauma. 2013;30(11):981-997. doi: 10.1089/neu.2012.2699

47. Stein TD, Montenigro PH, Alvarez VE, et al. Beta-amyloid deposition in chronic traumatic encephalopathy. Acta Neuropathol. 2015;130 (1):21-34. doi: 10.1007/s00401-015-1435-y

48. Omalu BI, DeKosky ST, Minster RL, Kamboh MI, Hamilton RL, Wecht $\mathrm{CH}$. Chronic traumatic encephalopathy in a National Football League player. Neurosurgery. 2005; 57(1):128-134.

49. Omalu BI, DeKosky ST, Hamilton RL, et al. Chronic traumatic encephalopathy in a national football league player: part II. Neurosurgery. 2006; 59(5):1086-1092.

50. McKee AC, Stern RA, Nowinski CJ, et al. The spectrum of disease in chronic traumatic encephalopathy. Brain. 2013;136(Pt 1):43-64. doi: 10.1093/brain/aws307

51. Parker HL. Traumatic encephalopathy ('punch drunk') of professional pugilists. J Neurol Psychopathol. 1934;15(57):20-28.

52. Neubuerger KT, Sinton DW, Denst J. Cerebral atrophy associated with boxing. AMA Arch Neurol Psychiatry. 1959;81(4):403-408.

53. Hof PR, Bouras C, Buée L, Delacourte A, Perl DP, Morrison JH. Differential distribution of neurofibrillary tangles in the cerebral cortex of dementia pugilistica and Alzheimer's disease cases. Acta Neuropathol. 1992;85(1):23-30.

54. Jordan BD. Chronic neurologic injuries in boxing. Med Aspects Boxing. 1993;1:177-185.

55. Crane PK, Gibbons LE, Dams-O'Connor K, et al. Association of traumatic brain injury with late-life neurodegenerative conditions and neuropathologic findings. JAMA Neurol. 2016;73(9):1062-1069. doi: 10.1001/jamaneurol.2016.1948

56. McKee AC, Stein TD, Kiernan PT, Alvarez VE. The neuropathology of chronic traumatic encephalopathy. Brain Pathol. 2015;25(3):350-364. doi: $10.1111 / \mathrm{bpa} .12248$

57. Kirov I, Whitlow C, Zamora C. Susceptibility-weighted imaging and magnetic resonance spectroscopy in concussion. Neuroimaging Clinics of North America 2018; 28(1): 91-105. doi:10.1016/j. nic.2017.09007

58. FDA authorizes marketing of first blood test to aid in the evaluation of concussion in adults. US Food and Drug Administration. February 13 , 2018. https://www.fda.gov/NewsEvents/Newsroom/ PressAnnouncements/ucm596531.htm. Accessed August 4, 2020.

59. Kyratzi E, Pavlaki M, Stefanis L. The S18Y polymorphic variant of $\mathrm{UCH}-\mathrm{L} 1$ confers an antioxidant function to neuronal cells. Hum Mol Genet. 2008;17(14):2160-2171. doi: 10.1093/hmg/ddn115

60. Wilkinson KD. Regulation of ubiquitin-dependent processes by deubiquitinating enzymes. FASEB J. 1997;11(14):1245-1256.

61. Roessmann U, Velasco ME, Sindely SD, Gambetti P. Glial fibrillary acidic protein (GFAP) in ependymal cells during development: an immunocytochemical study. Brain Res. 1980;200(1):13-21.

62. Stewart M, Quinlan RA, Moir RD. Molecular interactions in paracrystals of a fragment corresponding to the alpha-helical coiled-coil rod portion of glial fibrillary acidic protein: evidence for an antiparallel packing of molecules and polymorphism related to intermediate filament structure. J Cell Biol. 1989;109(1):225-234 
63. Cullen DK, Simon CM, LaPlaca MC. Strain rate-dependent induction of reactive astrogliosis and cell death in three-dimensional neuronal-astrocytic co-cultures. Brain Res. 2007:1158:103-115.

64. Huang XJ, Glushakova O, Mondello S, Van K, Hayes RL, Lyeth BG. Acute temporal profiles of serum levels of UCH-L1 and GFAP and relationships to neuronal and astroglial pathology following traumatic brain injury in rats. J Neurotrauma. 2015;32(16):1179-1189. doi: $10.1089 /$ neu.2015.3873

65. Zoltewicz JS, Scharf D, Yang B, Chawla A, Newsom KJ, Fang L. Characterization of antibodies that detect human GFAP after traumatic brain injury. Biomark Insights. 2012;7:71-79. doi: 10.4137/BMI.S9873

66. Papa L, Brophy GM, Welch RD, et al. Time course and diagnostic accuracy of glial and neuronal blood biomarkers GFAP and UCH-L1 in a large cohort of trauma patients with and without mild traumatic brain injury. JAMA Neurol. 2016;73(5):551-560. doi: 10.1001/ jamaneurol.2016.0039

67. Wang DD, Bordey A. The astrocyte odyssey. Prog Neurobiol. 2008;86 (4):342-367. doi: 10.1016/j.pneurobio.2008.09.015

68. Metting Z, Wilczak N, Rodiger LA, Schaaf JM, van der Naalt J. GFAP and $\mathrm{S} 100 \mathrm{~B}$ in the acute phase of mild traumatic brain injury. Neurology. 2012;78(18):1428-1433. doi: 10.1212/WNL.0b013e318253d5c7
69. Marchi N, Cavaglia M, Fazio V, Bhudia S, Hallene K, Janigro D. Peripheral markers of blood-brain barrier damage. Clin Chim Acta. 2004;342(1-2):1-12.

70. Cocchia D, Michetti F, Donato R. Immunochemical and immuno-cytochemical localization of S-100 antigen in normal human skin. Nature. 1981;294(5836):85-87. doi: 10.1038/294085a0

71. Cherry JD, Stein TD, Tripodis $Y$, et al. CCL11 is increased in the CNS in chronic traumatic encephalopathy but not in Alzheimer's disease. PLoS One. 2017;12(9):e0185541. doi: 10.1371/journal. pone. 0185541

72. Al Nimer F, Thelin E, Nyström H, et al. Comparative assessment of the prognostic value of biomarkers in traumatic brain injury reveals an independent role for serum levels of neurofilament light. PLoS One. 2015;10(7):e0132177. doi: 10.1371/journal.pone.0132177

73. McCrea M. Standardized mental status testing on the sideline after sport-related concussion. J Athl Train. 2001;36(3):274-279.

74. Cerritelli F, Ruffini N, Lacorte E, Vanacore N. Osteopathic manipulative treatment in neurological diseases: systematic review of the literature. J Neurol Sci. 2016;369:333-341. doi: 10.1016/j.jns.2016.08.062

() 2020 American Osteopathic Association 